\title{
Calcein Release Assay to Measure Membrane Permeabilization by Recombinant Alpha-Synuclein
}

Sayan Dutta ${ }^{1,3, \#}$, Ben G. Watson", \#, Seema Mattoo ${ }^{2,3,4, *}$ and Jean-Christophe Rochet ${ }^{1,3, *}$

${ }^{1}$ Department of Medicinal Chemistry and Molecular Pharmacology, Purdue University, West Lafayette, IN 47907, USA; ${ }^{2}$ Department of Biological Sciences, Purdue University, West Lafayette, IN 47907, USA; ${ }^{3}$ Purdue Institute for Integrative Neuroscience, Purdue University, West Lafayette, IN 47907, USA; ${ }^{4}$ Purdue Institute of Inflammation, Immunology and Infectious Disease, Purdue University, West Lafayette, IN 47907, USA

*For correspondence: smattoo@purdue.edu; irochet@purdue.edu

\#Contributed equally to this work

[Abstract] Lipid membranes are involved in regulating biochemical and biological processes and in modulating the selective permeability of cells, organelles, and vesicles. Membrane composition, charge, curvature, and fluidity all have concerted effects on cellular signaling and homeostasis. The ability to prepare artificial lipid assemblies that mimic biological membranes has enabled investigators to obtain considerable insight into biomolecule-membrane interactions. Lipid nanoscale assemblies can vary greatly in size and composition and can consist of a single lipid monolayer, a bilayer, or other more complex assemblies. This structural diversity makes liposomes suitable for a wide variety of biochemical and clinical applications. Here, we describe a calcein dye leakage assay that we have developed to monitor phospholipid vesicle disruption by alpha-synuclein ( $\alpha \mathrm{Syn}$ ), a presynaptic protein that plays a central role in Parkinson's disease (PD). We present data showing the effect of adenylylation of aSyn on aSyn-mediated vesicle disruption as an example. This assay can be used to study the effect of mutations or post-translational modifications on aSyn-membrane interactions, to identify protein binding partners or chemical entities that perturb these interactions, and to study the effects of different lipids on the permeabilization activity of aSyn or any other protein.

Keywords: Alpha-synuclein, Calcein release, Liposome, Membrane permeability, Parkinson's disease

[Background] Liposomes are nanoscale lipid assemblies formed as a result of hydrophilic and hydrophobic interactions among lipid head groups and fatty acid side chains, respectively. These biological membrane-mimicking lipid bilayer assemblies are a useful tool for studying membrane interactions of protein biomolecules due to (i) the flexibility of modulating the diameter of the liposomes, and thus the membrane curvature; and (ii) control over the lipid composition (Ahmed et al., 2019; Siontorou et al., 2017). Due to their bilamellar structure, liposomes contain an intralumenal aqueous cavity within which water-soluble reagents can be encapsulated (Duzgunes et al., 2010). This property has been exploited in drug delivery approaches (Alavi et al., 2017) and in studies of membrane permeabilization (Gupta et al., 2013) and vesicle fusion (Duzgunes et al., 2010) involving the encapsulation of fluorescent, hydrophilic (membrane-impermeable) markers. 
Alpha-Synuclein (aSyn) is a presynaptic protein that interacts with negatively charged cellular membranes as part of its biological function (Pineda and Burre, 2017). aSyn interactions with membranes are also known to induce aggregation of the protein on the membrane surface (Ysselstein et al., 2015).This process is hypothesized to lead to a disruption of membrane integrity as a result of lipid extraction during the growth of aSyn aggregates on the bilayer (Ysselstein et al., 2017; Reynolds et al., 2011). Evidence suggests that membrane-induced aSyn aggregation coupled with vesicle disruption contributes to aSyn neurotoxicity in PD (Ysselstein et al., 2017).

aSyn-mediated vesicle permeabilization has been modeled using different biophysical methods, including assays designed to monitor the leakage of dyes from synthetic liposomes (Reynolds et al., 2011; lyer et al., 2019). The water-soluble fluorophore calcein is well suited for dye leakage assays because it undergoes self-quenching at high concentrations in the lumenal space of synthetic vesicles, but shows a dramatic increase in fluorescence emission upon dilution in the extra-liposomal buffer following membrane disruption (Figure 1). We and other labs have used a calcein leakage assay to characterize the vesicle permeabilization activity of wild-type (WT) aSyn and mutant or post-translationally modified forms of the protein (Sanyal et al., 2019), as well as to assess the effect of an aSyn-interacting protein on vesicle disruption (Ysselstein et al., 2017). Here, we describe the calcein leakage assay and present data obtained using this method showing the effect of adenylylation on aSyn-mediated vesicle disruption.

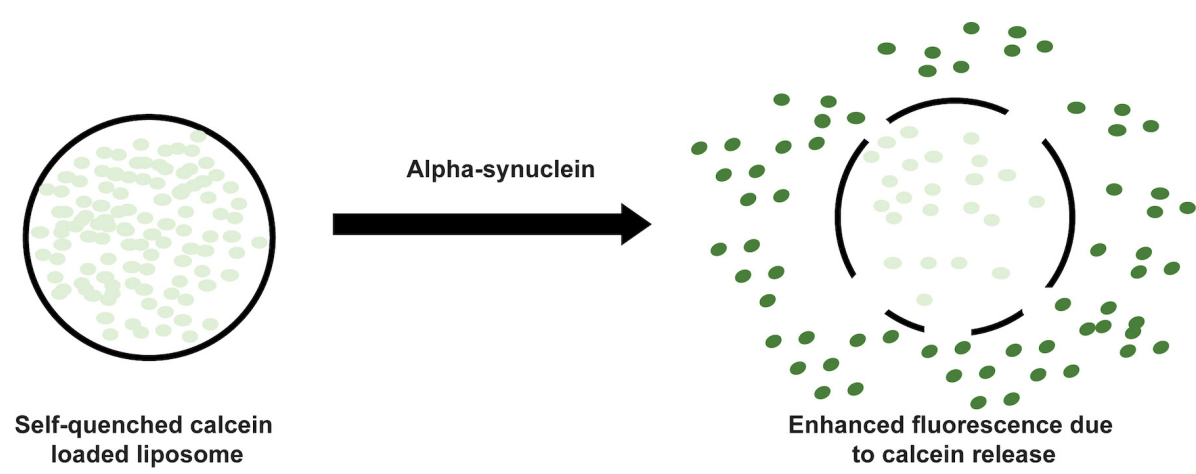

Figure 1. Schematic of calcein leakage assay designed to monitor liposome disruption in the presence of alpha-synuclein. Fluorescence increases over time, and the rate of increase is dependent upon the membrane permeabilization activity of the added alpha-synuclein variant.

\section{Materials and Reagents}

1. Eppendorf Safe-Lock Tubes (Eppendorf, catalog number: 022363204)

2. Solid black 96-well plate (Corning, catalog number: 07-200-336) or 384-well plate (Corning, catalog number: 09-761-21)

3. Plastic plate seal (Excel Scientific, catalog number: 100-SEAI-PLT)

4. Econo-Pac gravity-flow column (Bio-Rad, catalog number: 732-1010)

5. Polycarbonate membrane ( $0.05 \mu \mathrm{m}$, Avanti Polar Lipids, Inc., catalog number: 610003) 
6. Hamilton glass syringe (Avanti Polar Lipids, Inc., catalog number: 610036)

7. Glass vial (Sigma-Aldrich, Millipore Sigma, catalog number: 27023)

8. Glass round-bottom flask (Fisher Scientific, catalog number: 10-060-4A)

9. Disposable plastic cuvette

10. $0.22 \mu \mathrm{m}$ membrane

11. Egg phosphatidylglycerol (egg PG) (Avanti Polar Lipids, Inc., catalog number: 841138)

12. Egg phosphatidylcholine (egg PC) (95\%) (Avanti Polar Lipids, Inc., catalog number: 131601)

13. Calcein (Sigma-Aldrich, Millipore Sigma, catalog number: C0875)

14. Sephadex G-50 Superfine (GE Healthcare, Life Sciences, catalog number: 17004101 or 17004103)

15. Nitrogen gas (Indiana Oxygen, catalog number: 23-150224)

16. $\mathrm{NaOH}$ solid (Sigma-Aldrich, Millipore Sigma, catalog number: 221465)

17. $\mathrm{HCl}$ solution, $1 \mathrm{~N}$ (Fisher Scientific, catalog number: SA48-500)

18. $\mathrm{NaCl}$

19. $\mathrm{KCl}$

20. $\mathrm{Na}_{2} \mathrm{HPO}_{4}$

21. $\mathrm{KH}_{2} \mathrm{PO}_{4}$

22. Chloroform (Sigma-Aldrich, Millipore Sigma, catalog number: C2432)

23. Carbon-coated copper grid (Electron Microscopy Sciences, catalog number: CF400CU)

24. Nalgene rapid-flow sterile disposable filter unit (Fisher Scientific, catalog number: 09-740-43B)

25. $20 \%(\mathrm{v} / \mathrm{v})$ ethanol (Fisher Scientific, catalog number: A962S-4) in deionized $\mathrm{H}_{2} \mathrm{O}$

26. $0.02 \%(w / v) ~ \mathrm{NaN}_{3}$ (ACROS Organic, catalog number: AC190380050) in deionized $\mathrm{H}_{2} \mathrm{O}$

27. $1 \%(\mathrm{w} / \mathrm{v})$ Phosphotungstic acid (PTA) (Electron Microscopy Sciences, catalog number: 12501-23-4) in deionized $\mathrm{H}_{2} \mathrm{O}$

28. Bicinchoninic acid protein assay kit (Pierce, catalog number: 23227)

29. 1x PBS solution (prepared from 10x PBS stock, see Recipes)

30. $2 \mathrm{~N} \mathrm{NaOH}$ solution (see Recipes)

\section{Equipment}

1. Small beaker

2. Mini-Extruder (Avanti Polar Lipids, Inc., catalog number: 610020)

3. Filter support (Avanti Polar Lipids, Inc., catalog number: 610014)

4. Desiccator (Fisher Scientific, catalog number: 08-642-7)

5. Fisherbrand Analog Vortex Mixer (Fisher Scientific, catalog number: 02-215-414)

6. TECAN Infinite 200 Pro (TECAN, catalog number: INF-FPLEX)

7. OsmoPro Multi-Sample Micro-Osmometer (Fisher Scientific, catalog number: 22-046-680)

8. FEI Tecnai G2 20 transmission electron microscope (Thermo Fisher Scientific; Tecnai) 
9. Delsa Nano Submicron Particle Size and Zeta Potential Analyzer (Beckmann Coulter, catalog number: A54412AC)

10. Chemical fume hood

11. $-20^{\circ} \mathrm{C}$ freezer

\section{Software}

1. I-control $^{\mathrm{TM}}$ or Magellan ${ }^{\mathrm{TM}}$ Standard (used for calcein fluorescence data acquisition)

2. Gatan Digital Micrograph (Gatan Microscopy Suite) (used for electron micrograph acquisition)

3. Delsa ${ }^{\mathrm{TM}}$ Nano UI (used for dynamic light scattering data acquisition)

\section{Procedure}

A. Preparation of lipid/calcein solution

Note: This procedure uses chloroform, which should be handled in a chemical fume hood. Consult the chloroform safety data sheet for information about proper PPE, keeping in mind that chloroform is an eye, skin, and respiratory irritant and has been classified as a potential/probable human carcinogen.

1. Rinse one $25 / 50 \mathrm{ml}$ round-bottom flask (for each liposome preparation) sequentially with the following: soap water, deionized $\mathrm{H}_{2} \mathrm{O}$, acetone, and chloroform. Discard chloroform waste in a proper container. Oven dry the flask for $1-2 \mathrm{~h}$ or dry at $25^{\circ} \mathrm{C}$ overnight.

Note: Use a strong glass cleaning solution if the flask looks dirty.

2. Mix phosphatidylglycerol $(P G)$ and phosphatidylcholine $(P C)$ lipids in a 1:1 molar ratio so that the final (total) concentration of lipid is $5-10 \mathrm{mg} / \mathrm{ml}$ (or in a different molar ratio as needed). Lipids are obtained as a chloroform solution or a powder and should be stored as aliquots in a $-20^{\circ} \mathrm{C}$ freezer ( $200 \mu \mathrm{l}$ of a $25 \mathrm{mg} / \mathrm{ml}$ stock solution of egg PG or egg PC in each vial). ALWAYS store chloroform-lipid solutions in chloroform-compatible glass vials).

3. Remove any residual lipids from the glass vial by rinsing with chloroform; transfer the rinse to the round-bottom flask.

Note: The exact amount of chloroform used to rinse is not important, as the chloroform will be removed by evaporation in subsequent steps.

4. Use a stream of nitrogen or argon gas to evaporate the chloroform in the flask while swirling continuously to make a thin lipid film.

Note: Failure to continuously swirl the flask will result in an uneven distribution of lipids on the interior wall of the flask.

5. After the chloroform is evaporated, transfer the flask to a desiccator for $1 \mathrm{~h} \mathrm{minimum}$.

6. To prepare calcein-encapsulated vesicles (dye concentration, $103 \mathrm{mM}$ ), add $70 \mathrm{mg}$ of calcein to $865 \mu \mathrm{l}$ deionized $\mathrm{H}_{2} \mathrm{O}+135 \mu \mathrm{l} 2 \mathrm{~N} \mathrm{NaOH}$ in a $1.5 \mathrm{ml}$ microcentrifuge tube. Vortex the solution 
until all of the calcein is dissolved. Add the resulting $\sim 1 \mathrm{ml}$ calcein solution to the round-bottom flask with the dried lipids.

Note: If calcein is not dissolved, then $2 \mathrm{~N} \mathrm{NaOH}$ can be added dropwise (vortexing in between drops) until complete dissolution is achieved. The solution volume can be scaled down as needed to increase the liposome concentration. The osmolality must be kept identical (i.e., $\sim 280 \mathrm{mOsm} / \mathrm{kg}$ ) for both the intra- and extra-liposomal solutions at all stages and can be monitored using a standard micro-osmometer.

7. Swirl the flask for $30 \mathrm{~s}$ every $5 \mathrm{~min}$ over a period of $1 \mathrm{~h}$ to ensure that all lipids from the interior wall of the flask fall into the solution.

Note: The lipid suspension should be kept above the phase transition temperature of the lipid mixture (determined based on the guidelines of the supplier, in this case Avanti) during liposome preparation. A temperature of $25^{\circ} \mathrm{C}$ is acceptable for $1: 1$ (mol/mol) PG:PC. During this step, the chromatography column can be prepared (see Step D1).

\section{B. Extruder assembly}

1. Submerge 4 filter supports and 1 polycarbonate membrane into a small beaker of deionized $\mathrm{H}_{2} \mathrm{O}$.

2. Place the 2 internal membrane supports (Figure 2) on a flat surface with the O-rings facing up.

3. Place 2 soaked filter supports over the teflon orifice. The filter supports should adhere to the teflon orifice inside the O-ring inner diameter.

4. Insert the internal membrane support, with the filter support, into the extruder outer casing with the O-ring facing up.

5. Place 1 polycarbonate membrane in the extruder outer casing over the filter support and O-ring. The polycarbonate membrane is the thin, shiny disk; do not install one of the blue paper disks that separate the polycarbonate membranes. Depending on the desired liposome diameter, one can use membranes with a pore size that differs from the $0.05 \mu \mathrm{m}$ pore size mentioned in the Materials section.

Note: Polycarbonate membranes and filter supports are intended to be used for a single liposome preparation and should not be reused.

6. Place the remaining 2 soaked filter supports over the orifice of the remaining internal membrane support.

7. Carefully place the second internal membrane support into the casing (O-ring facing down), being careful not to twist the membrane support when it comes in contact with the polycarbonate membrane.

8. Place the teflon bearing into the retainer nut.

9. Place the retainer nut on the threaded end of the extruder outer casing, and tighten by hand until the retainer nut is finger tight; do not use a wrench or other tightening implement.

10. Fix the mini-extruder assembly into the extruder stand, keeping a vertex of the retainer nut facing up. 

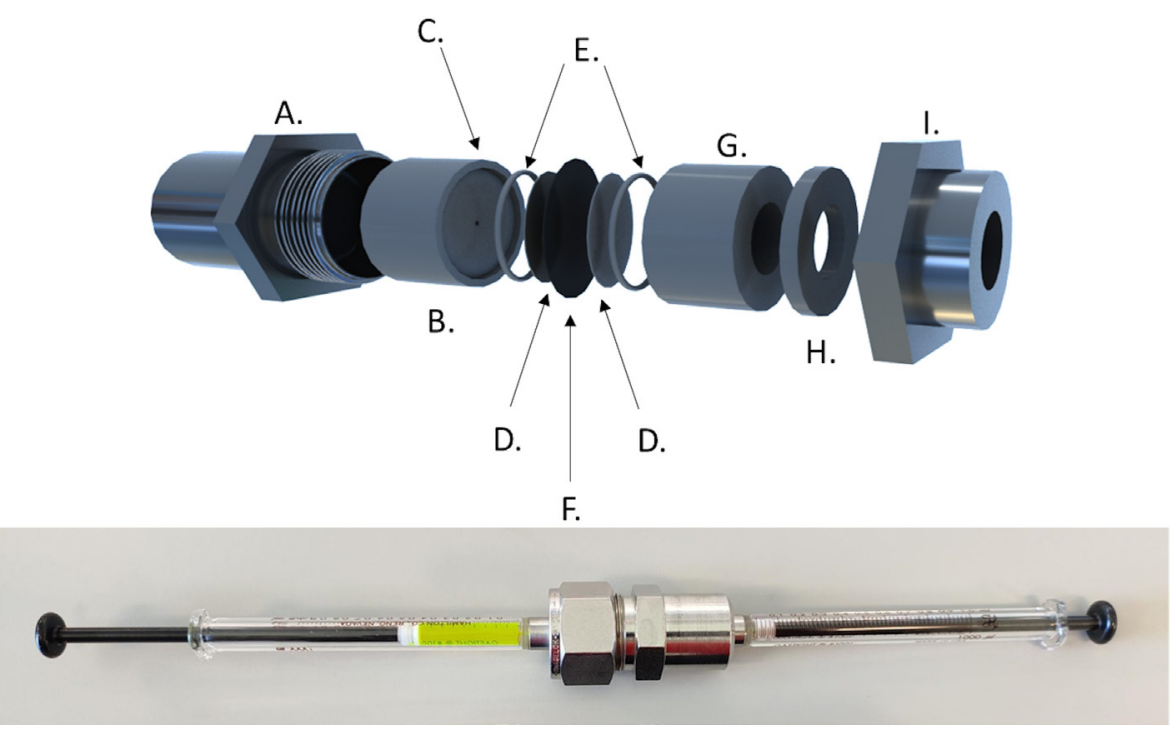

Figure 2. Assembly of extruder system. (Top) Schematic of the extruder assembly (adapted based on Avanti). A. Extruder outer casting. B. Internal membrane support. C. O-ring channel. D. Filter supports. E. O-rings. F. Polycarbonate membrane. G. Internal membrane support. H. Teflon bearing. I. Retainer nut. (Bottom) Syringe assembly with extruder.

C. Liposome preparation

1. Fill a clean Hamilton glass syringe with $\sim 1 \mathrm{ml}$ of lipid/calcein solution.

2. Carefully place the filled syringe into one end of the mini-extruder.

3. Carefully place an empty syringe into the opposite end of the mini-extruder. The plunger of the empty syringe should be depressed completely into the syringe barrel.

Note: The 2 syringes should be in line with each other, and parallel to the extruder stand.

4. Slowly depress the plunger of the filled syringe to pass the lipid/calcein solution through the membrane and support filters. The extruder stand should be held in place to prevent bending and to keep the syringes in line.

Note: Take note of the volume transferred into the previously empty syringe. This volume should be $\sim 100 \mu$ l less than the starting volume, but should remain constant throughout subsequent passages from one syringe to another. Further decreases in volume indicate leakage due to improper assembly.

5. Continue transfering the lipid/calcein solution from one syringe to the other for approximately 21 passes. The number of passes should always be odd, so that the solution collects in the initially empty syringe.

6. After $\sim 21$ passes, transfer the lipid/calcein solution into a $1.5 \mathrm{ml}$ microcentrifuge tube.

D. Separation of calcein-loaded liposomes from free dye

1. Resuspend $1 \mathrm{~g}$ of Sephadex $\mathrm{G}-50$ beads (or similar) in 8-10 $\mathrm{ml}$ deionized $\mathrm{H}_{2} \mathrm{O}$ in a small beaker, ensuring that the beads are completely covered. 
2. Mix the beads with continuous swirling, and transfer into an appropriately sized gravity-flow chromatography column with a bottom filter. Allow the resin to settle, and continue adding beads until the bed reaches a height of $7 \mathrm{~cm}$. Proper column height is crucial for optimal separation and flow rate. Tap the column to remove any bubbles and to allow the beads to settle. Place a filter on top of the beads. Store at $4{ }^{\circ} \mathrm{C}$ until ready for use. Columns may be stored in $20 \%(\mathrm{v} / \mathrm{v})$ ethanol (or $0.02 \%(\mathrm{w} / \mathrm{v}) \mathrm{NaN}_{3}$ for short periods) and reused.

Note: Before use, rinse the column with several column volumes (CV) of deionized $\mathrm{H}_{2} \mathrm{O}$ prior to exchanging to the desired mobile phase. Equilibrate the column with $>2 \mathrm{CV}$ of $1 \times \mathrm{PBS} / 0.02 \%$ $(w / v) \mathrm{NaN}_{3}$. Monitor flow to keep the column from running dry.

3. Pipette $\sim 300-500 \mu \mathrm{l}$ of lipid/calcein solution (Step C6) onto the top of the column bed, and monitor the separation of calcein bands (Figure 3). Calcein within liposomes should be a lighter shade of orange/yellow, whereas free calcein should be red.

Note: The loading volume will depend on the column's loading and separation capacity. After first applying the sample to the column, wait for the solution to enter the bed completely. Then, add the same volume of mobile phase, and let it fully enter as well. Next, apply a larger volume of mobile phase, and proceed with the separation until all visible bands have eluted from the column.

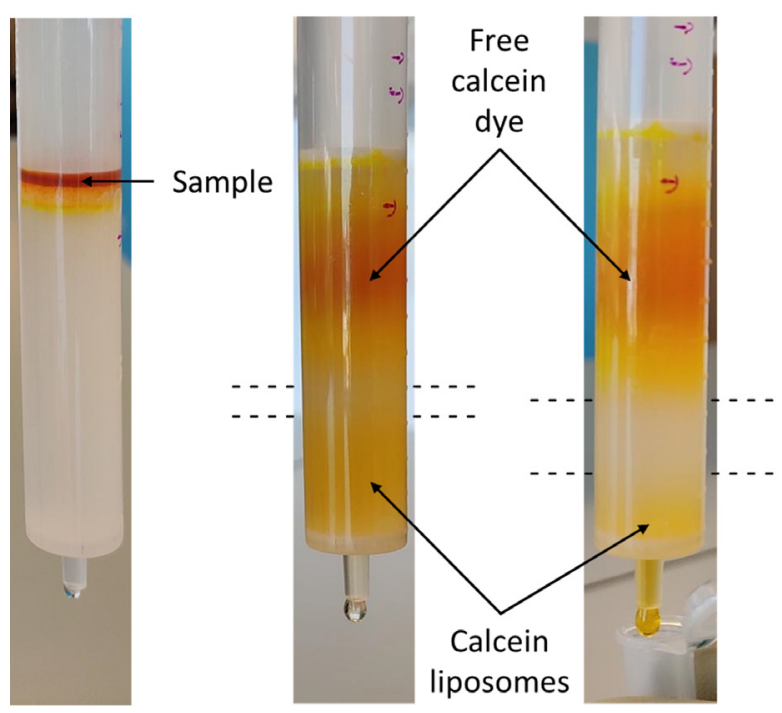

Figure 3. Separation of calcein-loaded liposomes from free dye using a Sephadex G-50 column. A mixture of calcein-loaded liposomes and unencapsulated dye is added slowly to the top of the separating column and given time to enter the bed. Buffer solution is slowly added to the top of the bed to drive the liposomes into the column. Liposomes appear as a distinct, light orange band ahead of the unencapsulated dye (red).

4. Collect the fraction containing the liposomes, which will elute ahead of the free calcein dye. Based on input and output volumes, calculate the dilution and the final concentration of the liposomes (in $\mathrm{mM}$ ). 
Note: Wash the column using excess buffer or deionized $\mathrm{H}_{2} \mathrm{O}$ until all of the free dye is removed. It is critical that the $\mathrm{pH}$ of the wash solution be 7.0 or greater to ensure complete removal of the dye. Store the column in $20 \%(\mathrm{~V} / \mathrm{V})$ ethanol until further use.

5. Store the liposomes at $4{ }^{\circ} \mathrm{C}$ until ready for use. Monitor the liposomes for stability by measuring their size distribution via dynamic light scattering (DLS) as well as the fluorescence of the liposome solution. The stability of the liposomes (and the optimal storage condition to ensure stability) may differ depending on their composition.

E. Liposome characterization

The morphology of the liposomes can be analyzed by negative stain transmission electron microscopy (TEM) and DLS.

\section{Negative stain TEM imaging:}

1. Discharge the grid using a glow discharge instrument (follow the instrument-specific protocol).

2. Add $3 \mu \mathrm{l}$ of a liposome solution (diluted to $0.5 \mathrm{mg} / \mathrm{ml}$ ) to a discharged grid, and incubate for 1 min at $25^{\circ} \mathrm{C}$.

3. Wash the sample with deionized $\mathrm{H}_{2} \mathrm{O}$ (without allowing the grid to dry completely).

4. Remove excess $\mathrm{H}_{2} \mathrm{O}$ by tapping the grid against filter paper, and stain with a $1 \%(\mathrm{w} / \mathrm{v})$ PTA solution $(3 \mu \mathrm{l})$ for $30 \mathrm{~s}$ to $1 \mathrm{~min}$.

5. Remove the excess solution by blotting with filter paper.

6. Image the sample using a transmission electron microscope (e.g., FEI Tecnai G2 20) operating at $200 \mathrm{KV}$.

\section{DLS:}

1. Dilute the liposome solution in 1x PBS (final concentration, $0.5 \mathrm{mg} / \mathrm{ml}$ ), and transfer into a reusable quartz cuvette or a disposable plastic cuvette, depending on the instrument. The volume examined will depend on the nature of the cuvette (e.g., for the experiment illustrated in Figure $4,80 \mu \mathrm{l}$ of a diluted liposome solution was transferred into a quartz cuvette).
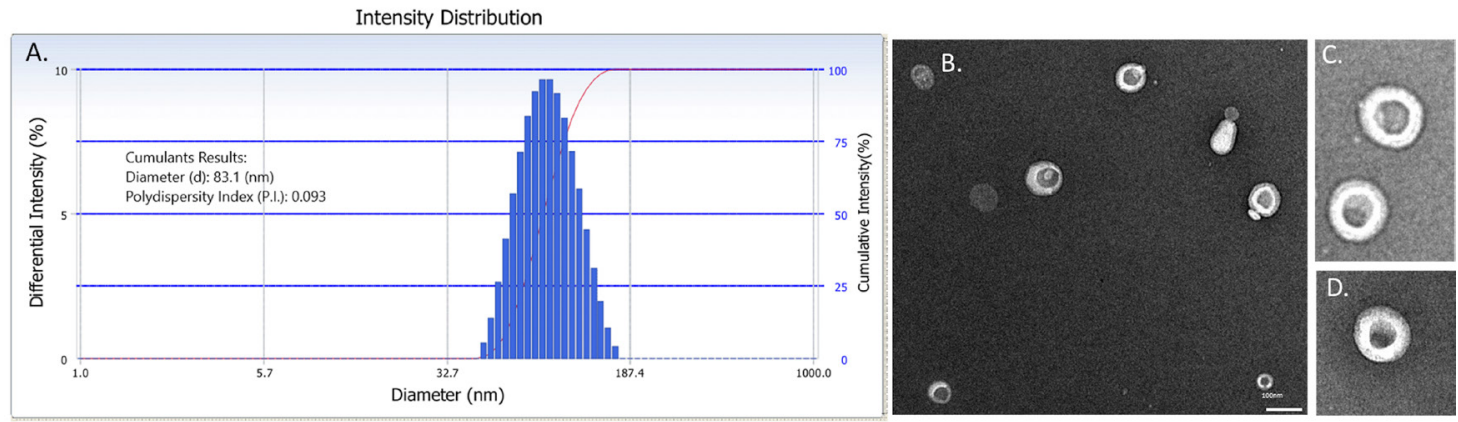

Figure 4. Characterization of liposomes via DLS and negative staining TEM. A.

Representative intensity distribution of liposomes in 1x PBS measured using a Delsa Nano instrument indicating a uniform distribution of liposomes with an average diameter of $83.1 \mathrm{~nm}$. 
B. Representative electron micrograph of liposomes showing the expected bilayer morphology. Scale bar represents $100 \mathrm{~nm}$. C-D. Manual zoom of individual liposomes.

2. Analyze the sample using a DLS instrument (e.g., Delsa Nano Submicron Particle Size and Zeta Potential Analyzer).

F. Monitoring calcein leakage

1. Calculate the volumes of $1 \times$ PBS, liposome stock solution, and aSyn stock solution required to set up an assay with a final volume of 50 or $100 \mu$. Final concentrations of liposomes and aSyn are $800 \mu \mathrm{M}$ and $40 \mu \mathrm{M}$, respectively. The aSyn protein used in this assay is purified from an $E$. coli expression system, and the concentration of aSyn stock solution is determined using a bicinchoninic acid protein assay kit, as described previously (Ysselstein et al., 2015 and 2017).

2. Add the assay components directly to the wells of a 96- or 384-well solid black plate, adding the protein last. Mix by gently pipetting half of the final volume up and down 3 to 5 times.

3. Cover the plate with a plastic plate seal, and transfer the plate into a microplate reader.

4. Set the microplate reader's excitation and emission wavelengths to $485 \mathrm{~nm}$ and $530 \mathrm{~nm}$, respectively. Collect fluorescence data at a defined time interval (e.g., every 15 or 30 min; Figure 5A).

\section{Data analysis}

\section{Lipid concentration calculation:}

Egg PG: $5 \mathrm{mg}$, egg PC: $5 \mathrm{mg}$ (mixed and added to $1 \mathrm{ml}$ of calcein solution)

Concentration before separation from free dye, $\mathrm{C}_{0}: 10 \mathrm{mg} / \mathrm{ml}$

Sample loaded onto Sephadex G-50 column: $0.4 \mathrm{ml}$

Output volume from column: $1.2 \mathrm{ml}$

Column dilution factor (DF): $1.2 \mathrm{ml} / 0.4 \mathrm{ml}=3$

Assuming no loss of liposome volume, the liposome/calcein concentration can be calculated as follows:

$$
\frac{C_{0}}{D F}=\frac{10 \mathrm{mg} / \mathrm{mL}}{3}=3.33 \mathrm{mg} / \mathrm{mL}=3.33 \mathrm{~g} / \mathrm{L}
$$

Average molecular weight of both egg PG and egg PC: $782 \mathrm{~g} / \mathrm{mol}$ (from Avanti product sheets). The molar concentration of lipids in the calcein-containing liposome solution is calculated as:

$3.33 \mathrm{~g} / \mathrm{L} \times 1 \mathrm{~mol} / 782 \mathrm{~g}=4.26 \times 10^{-3} \mathrm{~mol} / \mathrm{L}=4.26 \mathrm{mM}$

\section{Permeabilization calculations:}

Fractional permeabilization is calculated with the following equation: 


$$
\text { fractional permeabilization }=\frac{I_{t}-I_{0}}{I_{\max }-I_{0}}
$$

where $I_{t}$ is the fluorescence emission intensity at time $t, I_{0}$ is the intensity at time 0 , and $I_{\max }$ is the maximal intensity determined after detergent lysis of the vesicles.

The leakage kinetics, fractional permeabilization, and duration of the experiment are dependent on liposome composition and the biomolecule tested. Results can vary by approximately 10 to $20 \%$ because of differences among lipid batches and liposome preparations.

A representative curve showing the kinetics of aSyn-dependent calcein leakage is presented in Figure 5. These data are from a calcein leakage assay used to determine the effect of HYPE-dependent adenylylation/AMPylation on aSyn-mediated vesicle disruption, which was described previously (Sanyal et al., 2019). The assay revealed that adenylylation results in a perturbation of aSyn-membrane interactions, in turn leading to decreased membrane permeabilization evident from the reduced calcein fluorescence after $12 \mathrm{~h}$ (Figure 5).
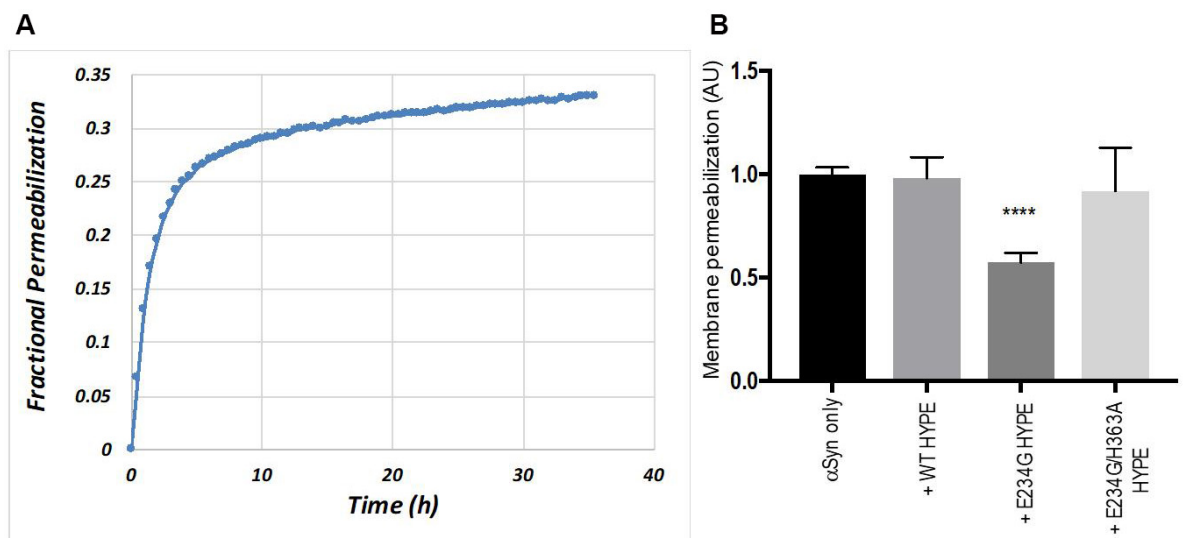

Figure 5. Adenylylation reduces the ability of aSyn to disrupt liposomes. A. Representative graph showing the kinetics of dye leakage from calcein-encapsulated liposomes (PG:PC, 1:1 mol/mol) incubated with human wild-type (WT) aSyn for $36 \mathrm{~h}$. B. Calcein-loaded liposomes were incubated with adenylylated aSyn (preincubated with constitutively active E234G-HYPE) or non-adenylylated aSyn (not preincubated with HYPE, or incubated with inactive forms of HYPE) and examined for an increase in calcein fluorescence (excitation and emission wavelengths, $485 \mathrm{~nm}$ and $530 \mathrm{~nm}$ ) (Sanyal et al., 2015 and 2019). The data are presented as relative membrane permeabilization after $12 \mathrm{~h}$, normalized with respect to non-adenylylated WT aSyn control. Mean \pm SEM, $n=4$ independent biological replicates (each with 3 technical replicates); ${ }^{* * *} P<0.0001$, E234G-HYPE versus each of the other groups, one-way ANOVA with Tukey's multiple comparisons post hoc test. 


\section{$\underline{\text { Recipes }}$}

1. 10x PBS solution (final volume, $1 \mathrm{~L}$ )

$80 \mathrm{~g} \mathrm{NaCl}$

$2 \mathrm{~g} \mathrm{KCl}$

$14.4 \mathrm{~g} \mathrm{Na}_{2} \mathrm{HPO}_{4}$

$2.4 \mathrm{~g} \mathrm{KH}_{2} \mathrm{PO}_{4}$

Solution brought to $\mathrm{pH} 7.4$ by adding $1 \mathrm{~N} \mathrm{HCl}$ or $2 \mathrm{~N} \mathrm{NaOH}$, then filtered through a $0.22 \mu \mathrm{m}$ membrane.

2. $2 \mathrm{~N} \mathrm{NaOH}$ solution (final volume, $0.5 \mathrm{~L}$ )

$40.0 \mathrm{~g} \mathrm{NaOH}$

\section{Acknowledgments}

We thank Dr. Daniel Ysselstein, Dr. Anwesha Sanyal, Dr. Omaima Ali, and Li-Kai Lin for their contributions at different stages during the development of the assay. We thank Dr. Robert Stahelin for permission to use the DLS instrument in his lab. We would also like to thank Jacob Glidden for designing the mini-extruder graphical representation.

This work was funded in part by grants from the National Institute of General Medical Sciences of the National Institute of Health (R01GM10092), the Indiana Clinical and Translational Research Institute (CTSI-106564), the Purdue Research Foundation (PRF-209104), and the Purdue Institute of Inflammation, Immunology, and Infectious Disease Core Start Grant (PI4D-209263) to SM; grants from the NINDS/NIH (R03NS108229) and the Branfman Family Foundation, as well as a Richard F. Borch Research Enhancement Award, to JCR; a Purdue Research Foundation Fellowship to JCR and SD; and an Eli Lilly-Stark Neuroscience Research Institute-CTSI pre-doctoral fellowship to SD.

\section{Competing interests}

The authors report no conflicts of interest.

\section{References}

1. Ahmed, K. S., Hussein, S. A., Ali, A. H., Korma, S. A., Lipeng, Q. and Jinghua, C. (2019). Liposome: composition, characterisation, preparation, and recent innovation in clinical applications. J Drug Target 27(7): 742-761.

2. Alavi, M., Karimi, N. and Safaei, M. (2017). Application of various types of liposomes in drug delivery systems. Adv Pharm Bull 7(1): 3-9. 
3. Duzgunes, N., Faneca, H. and Lima, M. C. (2010). Methods to monitor liposome fusion, permeability, and interaction with cells. Methods Mol Biol 606: 209-232.

4. Gupta, K., Jang, H., Harlen, K., Puri, A., Nussinov, R., Schneider, J. P. and Blumenthal, R. (2013). Mechanism of membrane permeation induced by synthetic beta-hairpin peptides. Biophys J 105(9): 2093-2103.

5. Iyer, A. and Claessens, M. M. A. E. (2019). Disruptive membrane interactions of alpha-synuclein aggregates. Biochim Biophys Acta Proteins Proteom 1867(5):468-482.

6. Pineda, A. and Burre, J. (2017). Modulating membrane binding of alpha-synuclein as a therapeutic strategy. Proc Natl Acad Sci U S A 114(6): 1223-1225.

7. Reynolds, N. P., Soragni, A., Rabe, M., Verdes, D., Liverani, E., Handschin, S., Riek, R. and Seeger, S. (2011). Mechanism of membrane interaction and disruption by alpha-synuclein. $J$ Am Chem Soc 133(48): 19366-19375.

8. Sanyal, A., Chen, A. J., Nakayasu, E. S., Lazar, C. S., Zbornik, E. A., Worby, C. A., Koller, A. and Mattoo, S. (2015). A novel link between Fic (filamentation induced by cAMP)-mediated adenylylation/AMPylation and the unfolded protein response. J Biol Chem 290(13): 8482-8499.

9. Sanyal, A., Dutta, S., Camara, A., Chandran, A., Koller, A., Watson, B. G., Sengupta, R., Ysselstein, D., Montenegro, P., Cannon, J., Rochet, J. C. and Mattoo, S. (2019). Alpha-synuclein Is a target of Fic-mediated adenylylation/AMPylation: possible implications for Parkinson's disease. J Mol Biol 431(12): 2266-2282.

10. Siontorou, C. G., Nikoleli, G. P., Nikolelis, D. P. and Karapetis, S. K. (2017). Artificial Lipid Membranes: Past, Present, and Future. Membranes (Basel) 7(3): 38.

11. Ysselstein, D., Dehay, B., Costantino, I. M., McCabe, G. P., Frosch, M. P., George, J. M., Bezard, E. and Rochet, J. C. (2017). Endosulfine-alpha inhibits membrane-induced alpha-synuclein aggregation and protects against alpha-synuclein neurotoxicity. Acta Neuropathol Commun 5(1): 3.

12. Ysselstein, D., Joshi, M., Mishra, V., Griggs, A. M., Asiago, J. M., McCabe, G. P., Stanciu, L. A., Post, C. B. and Rochet, J. C. (2015). Effects of impaired membrane interactions on alpha-synuclein aggregation and neurotoxicity. Neurobiol Dis 79: 150-163. 